\title{
Effects of Acute Creatine Monohydrate Supplementation on Arterial Stiffness and Muscle Oxygen Saturation in Young Men
}

\section{Colin J. Gimblet ${ }^{1}$, Thomas K. Pellinger ${ }^{2}$, John J. LaManca ${ }^{3}$, Austin T. Ortlip ${ }^{3}$, Morgan M. Vance ${ }^{3}$, Meghan Shepherd $^{3}$, Aaron T. Moore ${ }^{3}$, Timothy B. Staudmyer ${ }^{3}$, Zachary M. Townsend ${ }^{3}$, Timothy J. Werner ${ }^{3^{*}}$}

\author{
${ }^{1}$ Department of Health and Human Physiology, University of Iowa, Iowa City, USA \\ ${ }^{2}$ Department of Physical Therapy, University of Maryland Eastern Shore, Princess Anne, USA \\ ${ }^{3}$ Department of Exercise Science, Salisbury University, Salisbury, USA
}

Received Date: June 12, 2021; Accepted Date: June 22, 2021; Published Date: June 30, 2021

"Corresponding author: Timothy J. Werner, Salisbury University, Maggs Center 237A, 1101 Camden Ave, Salisbury, MD 21801, USA. Email: tjwerner@ salisbury.edu

\section{Abstract}

There are some important issues relating to the impact of acute creatine monohydrate supplementation on AS in the major elastic arteries and lower leg anterior compartment pressure. This study investigated the effects of acute creatine monohydrate supplementation on arterial stiffness (AS) and skeletal muscle oxygen saturation $(\mathrm{SmO} 2)$ in young men. A total of 20 male, physically active participants were randomized in a double-blind fashion to placebo (PL) $(n=10$, $22.9 \pm 3.1 \mathrm{yrs})$ or creatine $(\mathrm{CM})(\mathrm{n}=10,21.3 \pm 1.8 \mathrm{yrs})$ groups. Subjects received $0.3 \mathrm{~g} / \mathrm{kg} / \mathrm{day}$ creatine monohydrate or placebo in gelatin capsules for 7 days. Ultrasonography of the carotid artery, applanation tonometry, submaximal exercise tests (10-minute treadmill activity at $3.7 \mathrm{mph}$ and $9 \%$ incline), and $\mathrm{SmO} 2$ measurements were conducted at baseline and on day 7 of the study period. There was a significant time effect $(\mathrm{P}<0.05)$ in the $\mathrm{CM}$ group on central systolic blood pressure (cSBP) $(130 \pm 22 \mathrm{mmHg}$ to $112 \pm 38 \mathrm{mmHg})$, central pulse pressure (cPP) $(64 \pm 21 \mathrm{mmHg}$ to $58 \pm 21 \mathrm{mmHg})$ and arterial compliance (AC) $(1.09 \pm 0.35 \mathrm{~mm} 2 / \mathrm{mmHg} \times 10-1$ to $0.86 \pm 0.85$ $\mathrm{mm} 2 / \mathrm{mmHg} \times 10-1)$. Using a randomly controlled, doubleblind trial, acute creatine supplementation does appear to impact vascular compliance or oxygen saturation in skeletal muscle in young, healthy males.

Keywords: Beta-stiffness index, Central arterial blood pressure, Compartment pressure, Pulse wave velocity

\section{Introduction}

Cardiovascular disease (CVD) remains the leading cause of disability, morbidity, and death in modern societies [1]. Recently, a large emphasis has been placed on arterial stiffening and its role as an independent predictor for future cardiovascular events [2]. Arterial stiffening is composed of structural and dynamic components. The structural component is represented by the ratio of collagen and elastin fibers in the arterial media; while the dynamic component is represented by the tone of smooth muscles, dependent on vasoactive substances regulated by the endothelium [3]. There are several techniques and devices used to assess vessel physiology [4]. Carotid femoral pulse wave velocity (C-F PWV) is considered to be the gold standard measurement of arterial stiffness. Propagation of the pulse wave is inversely related to the distensibility of the arterial tube [2]. An increase in pulse wave velocity (PWV) by $1.0 \mathrm{~m} / \mathrm{s}$ was associated with a $14 \%$ increase in risk for cardiovascular events [5]. Beta-stiffness index is a common marker of local stiffness of the carotid artery and is considered a blood pressure independent index of central artery stiffness [4]. Carotid stiffness may be of particular interest, due to its susceptibility to the development of atherosclerosis [2]. Consequently, it is important to characterize interventions influencing vessel stiffness, to attenuate the progression of CVD.

Creatine supplementation has been popularized to athletes as an ergogenic aid to increase fat-free mass, muscular strength, and anaerobic performance [6]. The initial large dose, known as a loading dose, increases intramuscular phosphocreatine stores, aiding in the synthesis of adenosine triphosphate (ATP) and enhancing adaptation to exercise by augmenting growth factor expression [7]. Numerous studies have examined creatine supplementation and concluded it improves performance of shortterm high-intensity exercise [8]. Due to the commercialization of creatine as a performance aid, it is important to examine its physiological effects on the vasculature and arterial stiffness.

Currently, there are a limited number of studies examining the effect of acute creatine supplementation on the vasculature and measures of arterial stiffness. Moreover, those studies employed varied dosages and techniques, leaving questions regarding the impact of acute creatine supplementation on vascular stiffness. When given a loading dose of creatine $(0.3 \mathrm{~g}$ $\mathrm{x} \mathrm{kg} /$ day) for seven days, short-term creatine supplementation was shown to have no significant effect on resting heart rate or blood pressure in healthy men [9]. Similarly, seven days of supplementation (20g/day) showed no significant differences between creatine and placebo group in systolic blood pressure, diastolic blood pressure, mean arterial pressure, heart rate, and rate pressure product during exercise and rest [10]. Interestingly, a three-week creatine intervention (10g/day) was found to attenuate increases in brachial-ankle PWV (B-A $\mathrm{PWV}$ ) in response to exercise [11]. Although B-A PWV is a relevant measure of systemic vascular stiffness, it does not accurately represent central stiffness [4]. In contrast, seven days of creatine supplementation (21g/day) did not influence C-F PWV, blood pressure, or peripheral PWV (P PWV) in response to high intensity single leg knee extension in young, healthy subjects [12]. However, the methods used were dependent on blood pressure, thus results are subject to interpretation. Both studies found no significant difference between groups in resting measures of arterial stiffness before and after creatine supplementation $[\mathbf{1 1}, \mathbf{1 2}]$.

Another concern of creatine supplementation is its potential influence on intracellular fluid retention and thus compartment pressure in the anterior compartment muscles of the leg. This rise in pressure could negatively impact blood flow by compressing blood vessels in the area. Under non- 
supplemental conditions, anterior compartment muscle volume can increase by $20 \%$ during exercise [13]. Creatine supplementation also has been found to increase intracellular fluid by 2-3\% [14]. Coupled together, this augmented pressure could theoretically create an ischemic environment within the osteofascial compartment, resulting in clinical and subclinical related issues such as cramping, tightness, and/or pain within the anterior compartment muscle group. Multiple studies have found acute and chronic creatine supplementation increases anterior compartment pressure at rest and post exercise [1517]. However, they utilized an invasive and painful procedure that requires medical supervision, the Stryker Intracompartmental Pressure Monitor System, to record pressure changes. We proposed using Near Infrared Spectroscopy (NIRS), an indirect, noninvasive measurement of muscle oxygenation to evaluate compartmental muscle changes during the trial. NIRS devices have been used in the diagnosis of compartmental syndrome in the past [18], thus warranting further investigation. We hypothesized a 7-day administration of creatine would not have any positive or negative impact on indices of arterial stiffness. Additionally, we hypothesized the 7-day supplementation period would not reduce muscle oxygenation in the compartmental muscle group.

\section{Methods}

\section{Subjects}

A total of 20 subjects, aged 18-30, participated for this study from a pool of 24 students from Salisbury University that were initially screened. Participants were recruited from email advertisements and flyers placed around campus. Because of the potential for sex-related differences, only males were selected for the study. Previous investigations have found baseline and exercise training differences in parameters of arterial stiffness between males and females [19-23]. All participants were normotensive, and free from overt acute and chronic diseases. None of the participants were taking any medications or supplements known to influence hemodynamic properties or metabolic rate. All participants maintained a stable weight $( \pm 2 \mathrm{~kg})$ for at least 26 weeks prior to starting the study. They all reported to be involved in weight training for at least a year. There were no baseline differences between groups (Table 1). Participants completed a health history questionnaire and provided written consent before joining the study. The Salisbury University Institutional Review Board approved all aspects of the study.

\section{Study Design and Protocol}

Participants undertook a series of baseline measurements and tests including blood pressure acquisition, arterial tonometry, body composition, a treadmill walk test and a VO2max test. After completing the initial testing, subjects were randomly assigned in double-blind fashion to PL $(n=10)$ or CM $(n=10)$ by a faculty member not affiliated with the study. Both groups were instructed not to alter their normal diet and physical activity levels throughout the study period. Subjects received $0.3 \mathrm{~g} / \mathrm{kg} /$ day of creatine monohydrate (Pro Performance, GNC, Pittsburg, Pennsylvania) or placebo (Nutrasense, Lenexa, Kansas) of equal volume in gelatin capsules for seven days. They were instructed to consume creatine or the placebo with a glass of water at the same time every day. These protocols were similar to ones used in previous investigations $[\mathbf{1 5}, \mathbf{1 6}]$. All tests were completed within two days before and within one day after the trial period and the same testing sequence was followed for every subject. Subjects reported to the Salisbury University Human Physiology Laboratory between the 0800 to $1100 \mathrm{hrs}$ after a 12-hour fast for vascular assessments and body composition measurements. All exercise tests and pain assessments were completed in the Salisbury University Devilbiss Hall laboratory between 0900 to $1400 \mathrm{hrs}$. They were instructed to avoid tobacco and caffeinated products $12 \mathrm{hrs}$ prior, and alcohol consumption and moderate-to-vigorous exercise $24 \mathrm{hrs}$ prior to assessments.

\section{Procedures}

Pulse Wave Velocity. A tonometer (Complior Analytic Tonometer, Alam Medical, Vincennes, France) was used to obtain arterial pressure waveforms in the carotid, radial and femoral arteries and the average of ten cardiac cycles were recorded. Distances between the carotid and femoral sites, and carotid and radial sites were measured to the nearest $0.5 \mathrm{~cm}$ using an enlarged caliper (Hagl of, Madison, MS).PWV for the carotid-radial (C-R PWV) and carotid-femoral (C-F PWV) measurements were determined by dividing the distance between recording sites by the waveform foot-to-foot time delay to the $(\mathrm{PWV}=\mathrm{D}(\mathrm{m}) / \Delta \mathrm{t}(\mathrm{sec}))$. Participants rested in a supine position for at least 10 minutes prior to measurements and remained in this position for the duration of the tonometry session. Arterial Applanation Tonometry. The pressure waveform and wave amplitude in the right common carotid artery was assessed with a tonometer (Complior Analytic Tonometer, Alam Medical, Vincennes, France). Utilizing the transfer function, the pressure signal created by systolic blood pressure in the common carotid artery was calibrated to the mean arterial pressures measured in the brachial artery by automated sphygmomanometry. The transfer function calculated carotid systolic and diastolic blood pressure by using carotid waveforms along with systolic and diastolic pressures in the right brachial artery.

$\beta$ Stiffness Index. $\square$ stiffness index assesses the relationship between pressure and diameter changes in the carotid artery. It was calculated as: $\square=(\log \mathrm{P} 1 / \mathrm{P} 0) /((\mathrm{D} 1-\mathrm{D} 0) / \mathrm{D} 0)$, where $\mathrm{P} 0$ was the diastolic pressure and $\mathrm{P} 1$ was the systolic pressure in the carotid artery; and D0was the minimal diameter, and D1 was the maximal diameter of the carotid artery [24]. Right common carotid blood pressures were obtained from applanation tonometry and right common carotid diameters were measured with Doppler ultrasound, as described, below. $\square$ stiffness index was reported using arbitrary units. Stiffness index was a primary outcome in the present study because of its robust nature against significant changes in blood pressure.

\section{Carotid Artery Doppler Ultrasonography}

After 10minutes of rest in the supine position, right common carotid artery diameters were measured using a laptop Doppler ultrasound system(TerasonuSmart3300 NexGen, Burlington, Massachusetts)furnished with a linear ultrasound probe (Terason 15L4 Smart Mark, Burlington, Massachusetts). Bmode images of the common carotid artery were taken approximately $2 \mathrm{~cm}$ below the carotid bifurcation. Maximal and minimal diameters were measured during the cardiac cycle in triplicate. Diameters were measured as the distance from the media-intima interface of the near wall to the intimamedia interface of the far wall to the nearest $0.01 \mathrm{~cm}$.

\section{Resting Seated and Supine BP}

All blood pressure measurements were taken in a quiet, dimly lit, temperature-controlled $\left(21-22^{\circ} \mathrm{C}\right)$ laboratory. In accordance with the American Heart Association's guidelines for blood pressure acquisition (25), right brachial artery blood pressure measurements were assessed using an automated sphygmomanometer (Welch Allyn, New York) after resting in the seated or supine position for 10-minutes. Measurements were taken every two minutes and averaged when systolic and diastolic blood pressures were $\pm 6 \mathrm{mmHg}$ during three consecutive measurements.

\section{Body Mass and Composition}

Body height was assessed with a stadiometer (Detecto 439 Physician Beam Scale). Body weight was measured on a medical scale (Detecto 439 Physician Beam Scale) accurate to $+0.1 \mathrm{~kg}$ before breakfast and after voiding. Participants wore the same t-shirt and shorts at pre- and post-weigh ins. Fat mass and fat-free mass was measured using skin fold calipers (Lange, Beta Technology Inc., Cambridge, Maryland). Skin 
fold measurements were taken in duplicate on the right side from sites on the subscapular, midaxillary, triceps, chest, abdominal, suprailiac, and thigh. A third measurement was conducted if the first two measurements were $>2 \mathrm{~mm}$ from one another. A full explanation of the procedure is described elsewhere [26]. Body density, and thus body fat, was calculated from the summation of all the skinfold thicknesses using the seven-site formula for males [26].

\section{Maximal Exercise Test}

To determine subjects' VO2max, a graded maximal treadmill exercise test was performed approximately one week before collection of baseline measurements. All tests started with a 2-minute warm-up at 3.5 mphand $0 \%$ grade after which the speed was increased to $6 \mathrm{mph}$ and the incline was increased by $2 \%$ every 2 minutes until exhaustion. Expired gases were measured utilizing a Parvo Medics (Sandy, UT) metabolic cart. Heart rate was monitored utilizing a polar heart rate monitor (Polar Electro Inc., Woodbury, NY). And the 15-point Borg Scale was used to measure perceived exertion during each stage [27].

\section{Treadmill Walking Test and Muscle Oxygenation}

A treadmill walking test which has been utilized to validate NIRS as a tool in the diagnoses of chronic exertional compartment syndrome was performed 7-10 days after the VO2max test [18]. The subjects walked on a treadmill for 10 minutes at $6.0 \mathrm{~km} / \mathrm{h}$ with a $5 \%$ grade during which $\mathrm{SmO} 2$ was measured in the tibialis anterior muscle utilizing Near Infrared Spectroscopy (NIRS). The NIRS probe (Moxy, Hutchinson, $\mathrm{MN}$ ) was placed on the skin over the tibialis anterior muscle approximately $10 \mathrm{~cm}$ distal to the tibial tuberosity and $2 \mathrm{~cm}$ lateral to the tibial crest [18] and held in place using an elastic bandage. Data was continuously collected and visualized via Bluetooth technology and computerized data acquisition software (PeriPedal, Napoleon, IN). Baseline $\mathrm{SmO} 2$ was calculated by averaging $15 \mathrm{SmO} 2$ data points prior to the end of baseline collection taken prior to exercising. Peak exercise $\mathrm{SmO} 2$ was calculated by averaging $15 \mathrm{SmO} 2$ data points prior to the stop of exercise. Absolute change in $\mathrm{SmO} 2$ was equal to baseline $\mathrm{SmO} 2$ minus peak exercise $\mathrm{SmO}$. Finally, percentage change $\mathrm{SmO} 2$ was derived by dividing absolute change in $\mathrm{SmO} 2$ by baseline $\mathrm{SmO} 2$ and multiplying by100 [18].

\section{Statistical Analyses}

$\mathrm{G}^{*}$ Power software 3.1.9.7 was used to calculate a sample size with a priori for $\alpha=0.05$ and $\beta=0.80$ [28]. A sample of 20 individuals was determined for detecting significant differences with medium effect size (Cohen's $\mathrm{d}=0.3$ ). Statistical Package for the Social Sciences (IBM SPSS version 24, SPSS Inc., Chicago IL) computed descriptive and inferential statistics for all reported data. Unpaired Student's t-test was performed on baseline characteristics between the PL and CM groups. A 2 (time) x 2 (group) repeated measures analysis of variance (ANOVA) was used to examine the effects of treatment (creatine supplementation and placebo) and time (pre and post) interaction on arterial stiffness and other dependent variables. Pain threshold measurements were analyzed with a three-factor mixed design with repeated measure on two factors. Pain measures were taken pre- and post-exercise, and pre- and post-treatment. Tukey HSD post hoc analyses were performed on variables with significant Fratios. The data is expressed as means \pm standard deviation (SD).

\section{Results}

A total of 20 male subjects were recruited for this study. All subjects tolerated the creatine monohydrate and placebo well and reported no symptoms related to supplementation. Baseline and follow-up group characteristics are summarized in Table 1. Baseline characteristics were similar between the groups (all $\mathrm{P}>0.05$ ). Arterial stiffness variables at baseline and follow-up are listed in (Table 2). There was a baseline difference in cSBP (mean, $109 \pm 5 \mathrm{mHg} ; 95 \%$ confidence interval (CI) $105 \mathrm{mmHg}$ to $113 \mathrm{mmHg}$, vs. mean, $130 \pm 22$ $\mathrm{mmHg}$; $95 \%$ CI $114 \mathrm{mmHg}$ to $146 \mathrm{mmHg}, \mathrm{P}<0.05$ between the PL and CM groups, respectively) and arterial compliance (AC) (mean, $1.5 \pm 0.10 \mathrm{~mm} 2 / \mathrm{mmHg} \quad x \quad 10-1 ; 95 \% \quad$ CI $1.2 \mathrm{~mm} 2 / \mathrm{mmHg} \times 10$ - 1 to $1.8 \mathrm{~mm} 2 / \mathrm{mmHg} \times 10-1$, vs. mean, $1.1 \pm 0.10 \mathrm{~mm} 2 / \mathrm{mmHg} \times 10-1 ; 95 \%$ CI $0.8 \mathrm{~mm} 2 / \mathrm{mmHg} \times 10-$ 1 to $1.3 \mathrm{~mm} 2 / \mathrm{mmHg} \times 10-1, \mathrm{P}<0.05$ between the PL and $\mathrm{CM}$ groups, respectively). There were no other significant baseline differences in arterial stiffness indices (all P>0.05). Exercise response and $\mathrm{SmO} 2$ data are reported in Table 3. There were no baseline differences in these variables (all $\mathrm{P}>0.05$ ).

As shown in (Table 1), following the intervention there was a significant increase in fat free mass in the CM group only(pre-mean, $68.5 \pm 7.5 \mathrm{~kg} ; 95 \%$ CI $62.1 \mathrm{~kg}$ to $72.9 \mathrm{~kg}$, to post-mean $69.3 \pm 7.5 \mathrm{~kg}$; $95 \%$ CI $62.9 \mathrm{~kg}$ to $73.6 \mathrm{~kg}, \mathrm{P}<0.05$, effect size $(E S)=0.52$ ). There were no significant changes in subject characteristics reported between the groups (all $\mathrm{P}>$ 0.05). The CM group experienced significant reductions in cSBP (pre-mean, $130 \pm 22 \mathrm{mmHg}$; 95\% CI $114 \mathrm{mmHg}$ to 146 $\mathrm{mmHg}$, to post mean, $112 \pm 38 \mathrm{mmHg}$; $95 \% \mathrm{CI} 100 \mathrm{mmHg}$ to $126 \mathrm{mmHg}, \mathrm{P}<0.05, \mathrm{ES}=0.58)$, central pulse pressure (cPP) (pre-mean, $64 \pm 21 \mathrm{mmHg}$; $95 \%$ CI $51 \mathrm{mmHg}$ to $77 \mathrm{mmHg}$, vs. post-mean, $58 \pm 21 \mathrm{mmHg}$; $95 \% \mathrm{CI} 45 \mathrm{mmHg}$ to $71 \mathrm{mmHg}$, $\mathrm{P}<0.05, \mathrm{ES}=0.29$ ), and AC (pre-mean, $1.09 \pm 0.35 \mathrm{~mm} 2 / \mathrm{mmHg} \mathrm{x}$ $10-1 ; 95 \%$ CI $0.87 \mathrm{~mm} 2 / \mathrm{mmHg}$ x $10-1$ to $1.31 \mathrm{~mm} 2 / \mathrm{mmHg}$ x $10-1$, to post mean, $0.86 \pm 0.85 \mathrm{~mm} 2 / \mathrm{mmHg} \times 10-1 ; 95 \% \mathrm{CI}$ $0.33 \mathrm{~mm} 2 / \mathrm{mmHg} \times 10-1$ to $1.39 \mathrm{~mm} 2 / \mathrm{mmHg} \times 10-1, \mathrm{P}<0.05$, $\mathrm{ES}=0.35$ ). As seen in (Table 2, and Figures 1 and 2), there were no other significant changes in arterial stiffness variables following the intervention (all $\mathrm{P}>0.05$ ). Average exercise heart rate was significantly increased in the $\mathrm{CM}$ grouponly(mean, $151 \pm 18 \mathrm{bpm} ; 95 \% \mathrm{CI} 134 \mathrm{bpm}$ to $164 \mathrm{bpm}$, to mean, $154 \pm 17$ bpm; 95\% CI 141bpm to 166 bpm; $\mathrm{P}<0.05$, ES = 0.63). Percent change in $\mathrm{SmO} 2$ was significantly reduced in the CM group only (mean, $66.5 \pm 30.54 \%$; $95 \%$ CI $35.9 \%$ to $88.5 \%$, tomean $58.4 \pm 23.9 \%$; $95 \%$ CI $36.6 \%$ to $80.1 \%$, respectively; $\mathrm{P}<0.05$, $\mathrm{ES}=0.93)$. There were no other significant changes in performance-related and $\mathrm{SmO} 2$ variables following the intervention listed in Table 3 (all $\mathrm{P}>0.05$ ).

Following the intervention there were no significant changes in average pain levels between the groups (all $\mathrm{P}>0.05$ ). There was a non-significant reduction in percent change in C-F PWV (Figure 1) and no significant difference in percent change in C-R PWV (Figure 1) (all P>0.05). There were no significant changes to report in percent change in $\beta$ stiffness index and arterial compliance (Figure 2) (all $\mathrm{P}>0.05$ ). 


\begin{tabular}{|c|c|c|c|c|}
\hline & \multicolumn{2}{|c|}{ Placebo $(\mathrm{N}=10)$} & \multicolumn{2}{|c|}{ Creatine Monohydrate $(\mathbf{N}=10)$} \\
\hline Variable & PRE & POST & PRE & POST \\
\hline Age, yr & $22.9 \pm 3.1$ & NA & $21.3 \pm 1.8$ & NA \\
\hline Height, $\mathrm{cm}$ & $177.4 \pm 7.1$ & NA & $176.7 \pm 9.1$ & NA \\
\hline Weight, kg & $74.9 \pm 13.0$ & $74.9 \pm 12.4$ & $80.2 \pm 11.5$ & $80.9 \pm 11.4$ \\
\hline BMI, $\mathrm{kg} / \mathrm{m} 2$ & $23.9 \pm 3.4$ & $23.9 \pm 3.2$ & $25.4 \pm 2.7$ & $25.7 \pm 2.5$ \\
\hline Body Fat, \% & $12.3 \pm 4.6$ & $12.2 \pm 4.7$ & $14.2 \pm 5.3$ & $13.8 \pm 5.4$ \\
\hline Fat Mass, kg & $9.7 \pm 5.2$ & $10.6 \pm 5.5$ & $11.8 \pm 6.0$ & $11.6 \pm 6.0$ \\
\hline Fat Free Mass, $\mathrm{kg}^{*}$ & $65.2 \pm 8.4$ & $65.3 \pm 7.9$ & $68.5 \pm 7.5$ & $69.3 \pm 7.5$ \\
\hline Resting HR, bpm & $56 \pm 10$ & $59 \pm 9$ & $57 \pm 8$ & $56 \pm 9$ \\
\hline Seated Systolic BP, mmHg & $122 \pm 8$ & $119 \pm 6$ & $122 \pm 6$ & $122 \pm 9$ \\
\hline Seated Diastolic BP, $\mathrm{mmHg}$ & $69 \pm 7$ & $69 \pm 7$ & $69 \pm 6$ & $68 \pm 8$ \\
\hline Seated MAP, mmHg & $87 \pm 7$ & $85 \pm 6$ & $86 \pm 5$ & $86 \pm 7$ \\
\hline Supine Systolic BP, mmHg & $117 \pm 7$ & $119 \pm 6$ & $121 \pm 7$ & $117 \pm 7$ \\
\hline Supine Diastolic BP, mmHg & $66 \pm 7$ & $66 \pm 6$ & $66 \pm 7$ & $64 \pm 6$ \\
\hline Supine MAP, $\mathrm{mmHg}$ & $83 \pm 6$ & $83 \pm 6$ & $84 \pm 6$ & $81 \pm 5$ \\
\hline VO2 $\max , \mathrm{ml} / \mathrm{kg} / \mathrm{min}$ & $51.2 \pm 5.8$ & $\mathrm{~N} / \mathrm{A}$ & $53.5 \pm 4.6$ & $\mathrm{~N} / \mathrm{A}$ \\
\hline Maximum HR, bpm & $193 \pm 7.7$ & N/A & $195 \pm 6.6$ & N/A \\
\hline RERmax & $1.1 \pm 0.06$ & N/A & $1.1 \pm 0.04$ & N/A \\
\hline \multicolumn{5}{|c|}{$\begin{array}{l}\text { Values expressed as mean } \pm \mathrm{SD} \text {. BMI, body mass index; HR, heart rate; } \\
\mathrm{BP} \text {, blood pressure; MAP, mean arterial pressure; } \mathrm{VO}_{2} \mathrm{max} \text {, maximum volume } \\
\text { of oxygen consumption; RERmax, maximal respiratory exchange ratio } \\
* \mathrm{P}<0.05 \text { Time Effect, } \mathrm{CM} \text { group }\end{array}$} \\
\hline
\end{tabular}

Table 1: Subject characteristics pre-/post- supplementation.

\begin{tabular}{|c|c|c|c|c|}
\hline & \multicolumn{2}{|c|}{ Placebo $(\mathrm{N}=10)$} & \multicolumn{2}{|c|}{ Creatine Monohydrate $(\mathrm{N}=\mathbf{1 0})$} \\
\hline Variable & PRE & POST & PRE & POST \\
\hline C-F PWV, m/s & $7.4 \pm 1.2$ & $6.9 \pm 1.2$ & $6.5 \pm 0.8$ & $6.2 \pm 1.0$ \\
\hline C-R PWV, m/s & $9.9 \pm 3.1$ & $9.5 \pm 2.4$ & $8.8 \pm 1.2$ & $8.5 \pm 1.9$ \\
\hline Central Systolic BP, mmHg $\ddagger^{*}$ & $109 \pm 5$ & $112 \pm 7$ & $130 \pm 22$ & $112 \pm 38$ \\
\hline Central Diastolic BP, mmHg & $66 \pm 7$ & $66 \pm 6$ & $66 \pm 7$ & $64 \pm 6$ \\
\hline Central MAP, mmHg & $81 \pm 6$ & $81 \pm 4$ & $87 \pm 10$ & $83 \pm 7$ \\
\hline Central PP, mmHgt* & $43 \pm 7$ & $46 \pm 10$ & $64 \pm 21$ & $58 \pm 21$ \\
\hline$\beta-S I, U$ & $5.7 \pm 1.9$ & $5.8 \pm 2.1$ & $6.8 \pm 2.4$ & $7.1 \pm 2.8$ \\
\hline $\mathrm{AC}, \mathrm{mm} 2 / \mathrm{mmHg} \times 10-1 \dagger^{*}$ & $1.49 \pm 0.43$ & $1.47 \pm 0.49$ & $1.09 \pm 0.35$ & $0.86 \pm 0.85$ \\
\hline \multicolumn{5}{|c|}{$\begin{array}{l}\text { Values expressed as mean } \pm \mathrm{SD} ; \mathrm{C} \text {, carotid; } \mathrm{R} \text {, radial; } \mathrm{F} \text {, femoral; } \mathrm{PWV}, \\
\text { pulse wave velocity; } \mathrm{BP} \text {, blood pressure; MAP, mean arterial pressure; PP, C } \\
\text { pulse pressure; } \beta \text {-SI, } \beta \text { stiffness index; AC, arterial compliance } \\
+\mathrm{P}<0.05 \text { Baseline differences } \\
* \mathrm{P}<0.05 \text { Time Effect, CM group }\end{array}$} \\
\hline
\end{tabular}

Table 2: Arterial stiffness variables pre-/post- supplementation.

\begin{tabular}{|c|c|c|c|c|}
\hline & \multicolumn{2}{|c|}{ Placebo $(\mathrm{N}=10)$} & \multicolumn{2}{|c|}{ Creatine Monohydrate $(\mathrm{N}=10)$} \\
\hline Variable & PRE & POST & PRE & POST \\
\hline Resting HR, bpm & $56 \pm 10$ & $59 \pm 9$ & $57 \pm 8$ & $56 \pm 9$ \\
\hline Average Exercise HR, bpm** & $149 \pm 13$ & $145 \pm 12$ & $151 \pm 18$ & $154 \pm 17$ \\
\hline Peak $\mathrm{SmO} 2, \%$ & $36.6 \pm 11.0$ & $36.8 \pm 16.9$ & $18.1 \pm 16.3$ & $21.3 \pm 11.5$ \\
\hline Percent Change $\mathrm{SmO} 2, \% *$ & $39.9 \pm 16.7$ & $38.5 \pm 26.9$ & $66.5 \pm 30.5$ & $58.4 \pm 23.9$ \\
\hline Values exp & 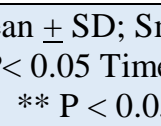 & $\begin{array}{l}\text { etal muscle } \\
\text { CM group } \\
\text { Effect }\end{array}$ & turation. & \\
\hline
\end{tabular}

Table 3: Exercise response and $\mathrm{SmO}_{2}$ variables pre-/post- supplementation. 


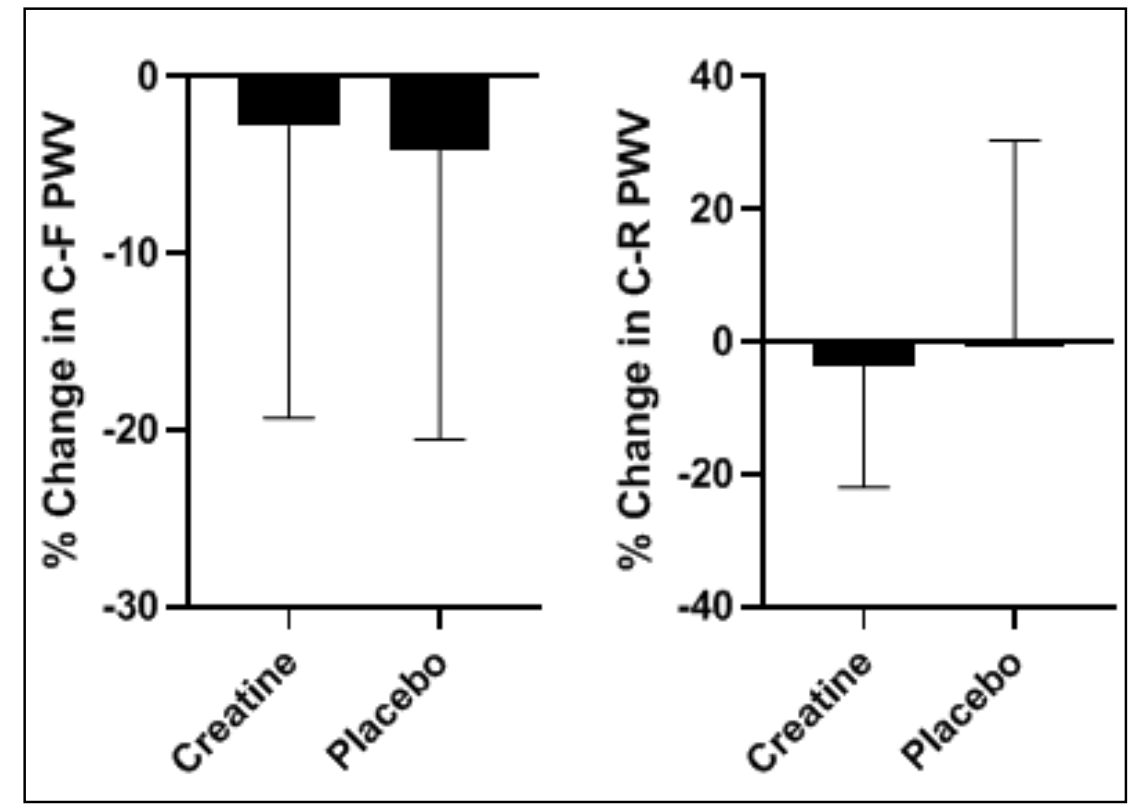

Figure1: The percent change in C-F PWV and C-R PWV following the intervention. $\mathrm{C}-\mathrm{F}=$ carotid femoral; $\mathrm{C}-\mathrm{R}=$ carotid radial; $\mathrm{PWV}=$ pulse wave velocity

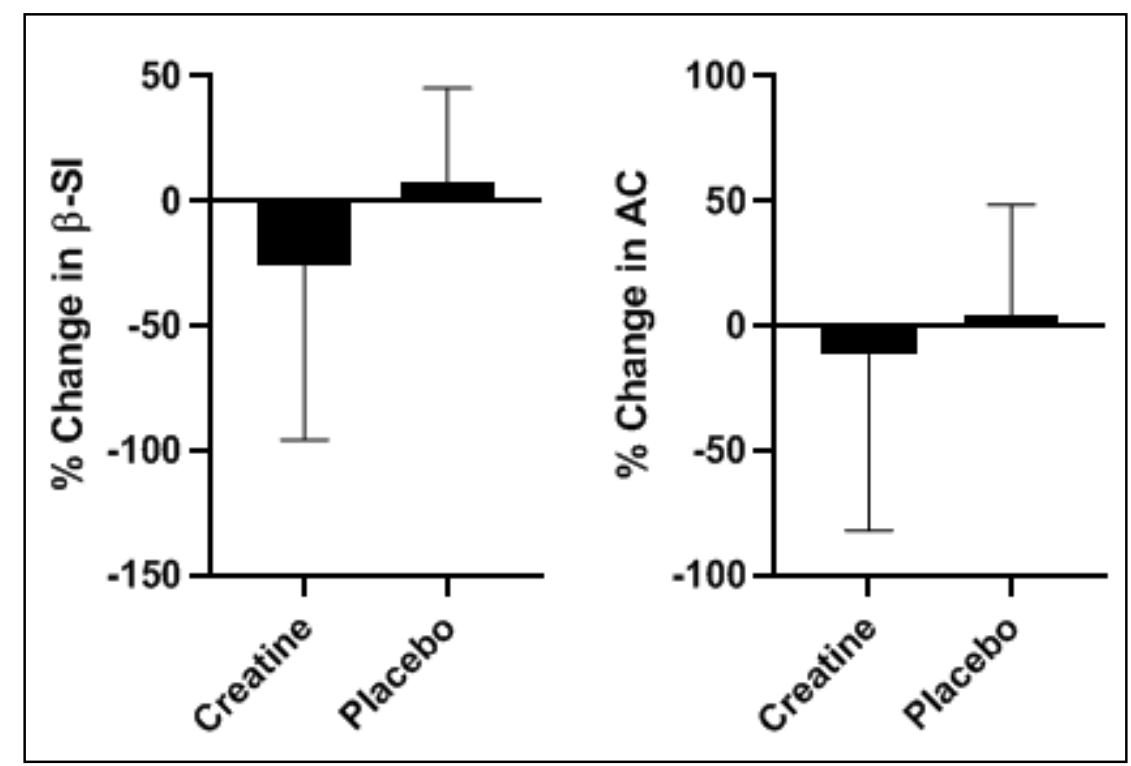

Figure 2: The percent change in $\beta$-SI and $\mathrm{AC}$ following the intervention. $\beta$-SI $=\beta$ stiffness index; $\mathrm{AC}=$ arterial compliance.

\section{Discussion}

In the present study, we compared vascular compliance and muscle oxygenation responses to an acute period of creatine supplementation in moderately active, healthy young men. The main findings of this study show creatine loading attenuatedcSBP, cPP, AC, and percent change in SmO2.Secondary findings report that creatine loading increased fat free mass and mean exercise heart rate. Both groups were similar in age and $\mathrm{VO} 2 \mathrm{max}$, indicating that fitness level and years of life were not a major influence on the observed changes. Together, these findings do not support our original hypothesis of an unaltered vascular compliance and muscle oxygenation response compared to healthy controls.

Very few studies have examined vascular changes in response to creatine loading. Among these, Sanchez-Gonzalez et al. [11] compared hemodynamic and arterial stiffness responses following a three-week trial in young, moderately active men. No significant absolute reductions were reported in blood pressure or arterial stiffness related variables. Similarly, Arazi et al. [10] were unable to detect changes in SBP, diastolic BP (DBP), and mean arterial blood pressure (MAP), at rest after one week of creatine loading. Utilizing a shorter dosing period (5 days), Mihic et al. (14) found no significant changes in measurements of peripheral BP. And finally, Aubry et al. [12] concluded creatine supplementation has no significant impact on resting hemodynamics after seven days of high-intensity resistance training. The present study confirms no changes to resting peripheral hemodynamic variables after a creatine loading period. Therefore, it appears creatine loading has little to no impact on resting left ventricular afterload. Since creatine loading increases muscle water content and not plasma volume, it would not be expected to significantly change resting $\mathrm{BP}[\mathbf{2 9}, \mathbf{3 0}]$.

Along the same lines, there was a group effect on average exercising HR, as the $\mathrm{CM}$ group experienced an overall increase, but no effect of time $(P=0.47)$. In comparison to our study, others have reported reductions in submaximal and maximal HR after a brief loading period through decreases in the muscle metaboreflex $[\mathbf{3 1}, \mathbf{3 2}]$. It is important to note that this was not a mechanistic study, thus rationale for these discrepancies are speculative. The differences could be explained by methodological differences, and/or fitness levels. Our subjects reported an average $\mathrm{VO} 2 \mathrm{max}$ of $51.2 \mathrm{ml} / \mathrm{kg} / \mathrm{min}$ and $53.5 \mathrm{ml} / \mathrm{kg} / \mathrm{min}$ in the PL and CM groups, respectively placing them in the 60th-80th percentile within ACSM's Cardio respiratory Fitness Classifications by Age and $\operatorname{Sex}(26)$.Previous studies also provided creatine in absolute amounts (i.e. $5-20 \mathrm{~g} / \mathrm{d}$ ) during the protocol. In contrast, our supplemental dose was relative to subjects' bodyweight (i.e. $0.3 \mathrm{~g} / \mathrm{kg} / \mathrm{d}$ ). In addition, average heart rate response was calculated for the entire treadmill exercise test whereas submaximal HR responses in the previous studies were not measured in aggregate, but rather reported from a given exercise intensity. Further research is necessary to uncover possible mechanism(s) of these conflicting results. 
We are the first to report significant reductions in cSBP, $\mathrm{cPP}$, and AC after a brief creatine loading period. cSBP and cPP are important vascular variables as they represent the true pressure against which the left ventricle works [2]. AC, a measurement of arterial extension and recoil, is determined by the volume-pressure relationship within the carotid artery. Blood vessels become stiffer when exposed to higher pressures and blood volumes, therefore, the attenuated pressures in the central arteries could have been enough to lower AC. Two other studies have found reductions in a pressure-dependent index of arterial stiffness. Percent change in brachial artery PWV (B PWV) was found to significantly decrease after a creatine loading phase [11]. However, B PWV can overestimate central PWV by $20 \%$, indicating that caution must be observed when interpreting these results. In a similar study, resting C-F PWV was left unchanged after a 7-day creatine loading cycle [12]. These studies examined the acute effects of isokinetic knee extension exercise on arterial stiffness, not necessarily a common exercise training modality. We also did not observe any significant changes in C-F PWV, the gold standard for assessing arterial stiffness [2], or $\beta$ stiffness index, a blood pressure-independent variable of arterial stiffness. Indeed, C-F PWV has an important clinical application as an increase of $1 \mathrm{~m} / \mathrm{s}$ is correlated with a $14 \%$ increase in relative risk of cardiovascular mortality [5]. Therefore, it is unlikely vascular compliance change as a result of acute creatine supplementation, indicating a minimal impact on cardiovascular risk profile.

We found a significant reduction in percent change in SmO2utilizing a non-invasive measurement of $\mathrm{SmO}$, indicating an improvement in muscle oxygenation during exercise following a creatine loading period (Table 3). Studies examining creatine supplementation have shown an increase in baseline and post exercise compartment pressure $[15, \mathbf{1 6}]$. Potteiger et al. [15] observed anterior compartment pressures after 7 and 35 days of creatine supplementation using direct pressure measurements and found greater levels of pressure compared to baseline. They also reported reductions in pressure only after 28 days of no supplementation [15]. Hile et al. [16] reported a moderate effect size in resting anterior compartment pressures after an acute loading period in well trained men. In theory an increase in compartment pressure could inhibit blood flow thus reducing exercise and recovery muscle oxygenation, however our data indicate that creatine supplementation may actually improve $\mathrm{SmO} 2$ during exercise.

One to two weeks of creatine supplementation has been shown to increase total body mass by approximately $1 \mathrm{~kg}$, predominately in the form of fat free mass $[14,33,34]$. Most of this weight gain is attributed to intra-myofibrillar water retention mediated by the sodium and amino acid concentration gradient across the sarcolemma created by creatine transportation into the muscle fiber [35]. The present study confirms these results.

We acknowledge potential limitations to the study. There is a high variability in creatine storage and utilization has been reported across age groups [36]. Additionally, the homogeneity of the subject population and the generalizability of these findings to females is limiting. Future studies should examine the interactions between acute creatine supplementation on a heterogeneous population including females and broader age ranges to uncover possible physiological differences. There were also various strengths related with the study protocol. The use of a double-blinded, placebo-controlled randomized model and measuring $\beta$ stiffness index to validate measurements of other arterial stiffness blood pressure-dependent indices were all important to improving our understanding of the true effects of creatine loading on arterial physiology. To our knowledge, no other study has examined this relationship.

In summary, the results of this study suggest a 7-day creatine supplementation period reduce arterial stiffness indices in young, trained males. These data are consistent with other investigations, suggesting larger dosages of creatine supplementation during the loading period have no negative outcomes on vascular compliance and compartment muscle blood flow. As such, creatine may be a supplementation strategy to improving athletic performance while having minimal to no impact on vascular physiology.

\section{Practical Applications}

The current findings support the use of acute creatine monohydrate supplementation in active, healthy young men. It does not appear that creatine monohydrate causes any negative impact on vascular compliance or muscle oxygen saturation. As such, the consumption of creatine loading dose appears to be safe, albeit acutely. Strength and Conditioning Specialists and Certified Personal Trainers should advise their clientele accordingly.

\section{Acknowledgements}

This research was supported by Salisbury University's Faculty Mini-Grant. The authors have no conflicts of interest to disclose. The authors would also like to thank all of the participants for volunteering in the study.

\section{References}

1. Seals DR, Kaplon RE, Gioscia-Ryan RA, LaRocca TJ (2014) you're only as old as your arteries: translational strategies for preserving vascular endothelial function with aging. Physiology (Bethesda) 29: 250-264.

2. Laurent S, Cockcroft J, Van Bortel L, Boutouyrie P, Giannattasio C, et al. (2006) Expert consensus document on arterial stiffness: methodological issues and clinical applications. European Heart Journal 27: 2588-2605.

3. Janic M, Lunder M, Sabovic M (2014) Arterial stiffness and cardiovascular therapy. Biomed Res Int 621437.

4. Tanaka H (2018) Various Indices of Arterial Stiffness: Are They Closely Related or Distinctly Different? Pulse (Basel) 5: 1-6.

5. Vlachopoulos C, Aznaouridis K, Stefanadis C (2010) Prediction of cardiovascular events and all-cause mortality with arterial stiffness: a systematic review and meta-analysis. J Am Coll Cardiol 55: 1318-27.

6. Kreider RB, Kalman DS, Antonio J, Ziegenfuss TN, Wildman R, et al. (2017) International Society of Sports Nutrition position stand: safety and efficacy of creatine supplementation in exercise, sport, and medicine. J Int Soc Sports Nutr 14:18.

7. Maughan RJ, Burke LM, Dvorak J, Larson-Meyer DE, Peeling P, et al. (2018) IOC consensus statement: dietary supplements and the high-performance athlete. $\mathrm{Br} \mathrm{J}$ Sports Med 52: 439-455.

8. Volek JS, Rawson ES (2004) Scientific basis and practical aspects of creatine supplementation for athletes. Nutrition 20: 609-614.

9. Volek JS, Mazzetti SA, Farquhar WB, Barnes BR, Gomez AL, et al. (2001) Physiological responses to short-term exercise in the heat after creatine loading. Med Sci Sports Exerc 33: 1101-1108.

10. Arazi H, Rahmaninia F, Hosseini K, Asadi A (2015) Effects of short term creatine supplementation and resistance exercise on resting hormonal and cardiovascular responses. Science and Sports 30:105-109.

11. Sanchez-Gonzalez MA, Wieder R, Kim JS, Vicil F, Figueroa A (2011) Creatine supplementation attenuates hemodynamic and arterial stiffness responses following an acute bout of isokinetic exercise. Eur J Appl Physiol 11: 1965-1971.

12. Aubry RL, Whinton AK, Burr JF (2018) The effect of creatine supplementation on the response of central and peripheral pulse wave velocity to high-intensity resistance exercise. Cogent Medicine 5: 1512352.

13. Wallensten R, Eklund B (1983) Intramuscular pressures and muscle metabolism after short-term and long-term exercise. Int J Sports Med 4: 231-235.

14. Mihic S, MacDonald JR, McKenzie S, Tarnopolsky MA (2000) Acute creatine loading increases fat-free mass, but 
does not affect blood pressure, plasma creatinine, or CK activity in men and women. Med Sci Sports Exerc 32: 291-296.

15. Potteiger JA, Carper MJ, Randall JC, Magee LJ, Jacobsen DJ, et al. (2002) Changes in Lower Leg Anterior Compartment Pressure Before, During, and After Creatine Supplementation. J Athl Train 37: 157-163.

16. Hile AM, Anderson JM, Fiala KA, Stevenson JH, Casa DJ, et al. (2006) Creatine supplementation and anterior compartment pressure during exercise in the heat in dehydrated men. J Athl Train 41: 30-35.

17. Potteiger JA, Randall JC, Schroeder C, Magee LM, Hulver MW (2001) Elevated Anterior Compartment Pressure in the Leg After Creatine Supplementation: A Controlled Case Report. J Athl Train 36: 85-88.

18. van den Brand JG, Verleisdonk EJ, van der Werken $C$ (2004) Near infrared spectroscopy in the diagnosis of chronic exertional compartment syndrome. Am J Sports Med 32: 452-456.

19. Mitchell GF, Parise H, Benjamin EJ, Larson MG, Keyes MJ, et al. (2004) Changes in arterial stiffness and wave reflection with advancing age in healthy men and women: the Framingham Heart Study. Hypertension 43: 123912345 .

20. Hayward CS, Kelly RP (1997) Gender-related differences in the central arterial pressure waveform. J Am Coll Cardiol 30: 1863-1871.

21. Perdomo SJ, Moody AM, McCoy SM, Barinas-Mitchell E, Jakicic JM, et al. (2016) Effects on carotid-femoral pulse wave velocity $24 \mathrm{~h}$ post exercise in young healthy adults. Hypertens Res 39: 435-439.

22. Doonan RJ, Mutter A, Egiziano G, Gomez YH, Daskalopoulou SS (2013) Differences in arterial stiffness at rest and after acute exercise between young men and women. Hypertens Res 36: 226-231.

23. Collier SR (2008) Sex differences in the effects of aerobic and anaerobic exercise on blood pressure and arterial stiffness. Gend Med 5: 115-123.

24. Nakamura T, Nakahara Y, Matsumuro A, Hata H (2004) [Assessment of carotid arterial distensibility using beta stiffness index in the diagnosis of arteriosclerosis]. Nihon Rinsho 3: 272-282.

25. Atherosclerosis $H$, Obesity in the Young Committee of the American Heart Association Council on Cardiovascular Disease in the Y, Alpert B, McCrindle B, Daniels S, Dennison B, et al. (2006) Recommendations for blood pressure measurement in human and experimental animals; part 1: blood pressure measurement in humans. Hypertension 48: e3; author reply e5.

26. American College of Sports Medicine, Riebe D, Ehrman JK, Liguori G, Magal M (2018) ACSM's guidelines for exercise testing and prescription. Tenth edition. ed. Philadelphia: Wolters Kluwer 472 pages $p$.

27. Dunbar CC, Robertson RJ, Baun R, Blandin MF, Metz K, et al. (1992) The validity of regulating exercise intensity by ratings of perceived exertion. Med Sci Sports Exerc 24: 94-99.

28. Faul F, Erdfelder E, Lang AG, Buchner A (2007) G*Power 3: a flexible statistical power analysis program for the social, behavioral, and biomedical sciences. Behav Res Methods 39: 175-191.

29. Gordon A, Hultman E, Kaijser L, Kristjansson S, Rolf CJ, et al. (1995) Creatine supplementation in chronic heart failure increases skeletal muscle creatine phosphate and muscle performance. Cardiovasc Res. 30: 413-418.

30. Tuganowski W (1996) The effects of $\mathrm{PCr}$ introduced simultaneously into myocardiac cells. European Journal of Physiology 431: 652-657.

31. Nelson AG, Day R, Glickman-Weiss EL, Hegsted M, Kokkonen J, et al. (2000) Creatine supplementation alters the response to a graded cycle ergometer test. Eur J Appl Physiol 83: 89-94.

32. Murphy AJ, Watsford ML, Coutts AJ, Richards DA (2005) Effects of creatine supplementation on aerobic power and cardiovascular structure and function. J Sci Med Sport 8: 305-313.

33. Earnest CP, Snell PG, Rodriguez R, Almada AL, Mitchell TL (1995) The effect of creatine monohydrate ingestion on anaerobic power indices, muscular strength and body composition. Acta Physiol Scand 153: 207-209.

34. Birch R, Noble D, Greenhaff PL (1994) The influence of dietary creatine supplementation on performance during repeated bouts of maximal isokinetic cycling in man. Eur J Appl Physiol Occup Physiol 69: 268-276.

35. Steenge GR, Lambourne J, Casey A, Macdonald IA, Greenhaff PL (1998) Stimulatory effect of insulin on creatine accumulation in human skeletal muscle. Am J Physiol 275: E974-979.

36. Rawson ES, Clarkson PM, Price TB, Miles MP (2002) Differential response of muscle phosphocreatine to creatine supplementation in young and old subjects. Acta Physiol Scand 174: 57-65.

Citation: Gimblet CJ, Pellinger TK, LaManca JJ, Ortlip AT, Vance MM, Shepherd M, Moore AT, Staudmyer TB, Townsend ZM, Werner TJ (2021) Manuscript Title: Effects of Acute Creatine Monohydrate Supplementation on Arterial Stiffness and Muscle Oxygen Saturation in Young Men. Adv Ortho and Sprts Med: AOASM-146. 